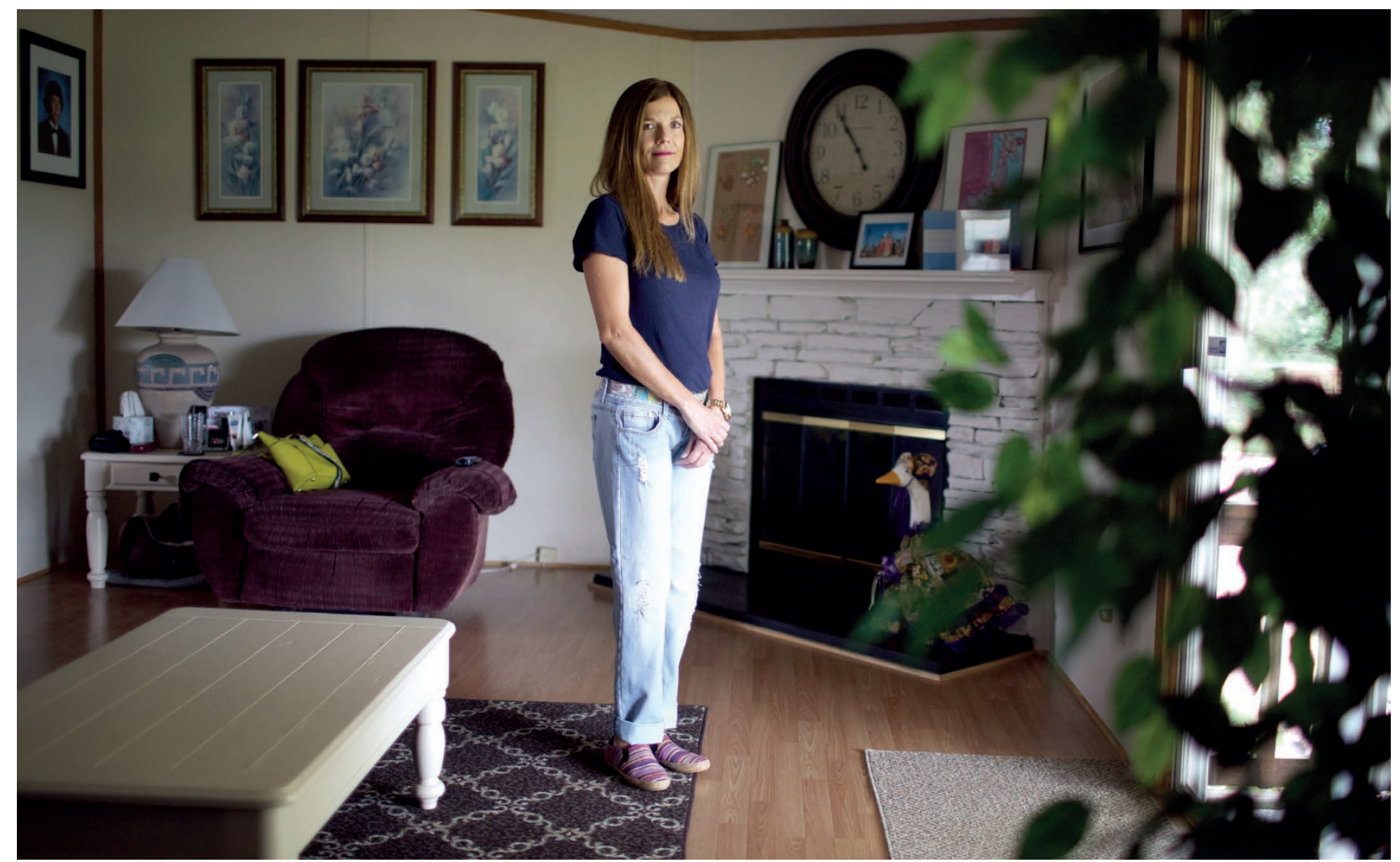

When Elizabeth Davis had her exome sequenced after 30 years of misdiagnosis, it revealed a genetic mutation and suggested an effective treatment.

\title{
DIAGNOSIS
}

\section{A clear answer}

\section{Living with a rare disease but no concrete diagnosis can be difficult. Genetic sequencing may finally provide a solution.}

\section{BY EMILY SOHN}

$\mathrm{E}$ lizabeth Davis began searching for answers at the age of six, when she suddenly started walking on her toes. With time came other mysterious symptoms, including foot pain, leg weakness, periodic weakness in her arms, and a muffled voice. Her knees knocked together when she walked, and her toes curled under her feet. By the time she reached adulthood, she was unable todo simple things such as shop for groceries on her own.

Davis saw plenty of doctors over the years. From the age of nine, orthopaedic specialists operated four times to lengthen the tendons in her heels. Neurologists took magnetic resonance imaging (MRI) scans of her brain and spine. But no one could identify her condition. In her thirties, one doctor suggested cerebral palsy, and she later got a diagnosis of hereditary spastic paraplegia, but no one was certain. "I just wanted to know 100\%," says Davis, now a 40-year-old mother of two boys in Goldsboro, North Carolina. "Nobody could tell me."

Finally, in 2012, Davis enrolled in a trial at the University of North Carolina at Chapel Hill, where scientists used a blood sample to sequence her exome - the portion of DNA that codes for functional genes. After 30 years of waiting, she finally learned the source of her problems: a mutation in a gene called $G C H 1$. The mutation suggested that she might respond to a drug called levodopa, which supplies the brain with dopamine and helps some patients with Parkinson's disease. Three days later, she was standing in a shop when she felt her toes uncurl. Two months later, she strolled into the clinic unaccompanied - the first time she had walked without crutches for 17 years.

Such stories provide hope that the next generation of gene sequencing has the potential to transform people's lives. Now more affordable than ever, the technology is making headway in diagnosing rare diseases, giving patients answers to medical mysteries that in some cases have dragged on for years.

But James Evans, a medical geneticist at the University of North Carolina, urges caution. The complete resolution of Davis's case makes her a rare exception in a field where there is still great uncertainty about how to interpret the meaning of small blips in vast stretches of genetic code. And even when clear diagnoses do emerge, doctors are usually still unable to do anything to help.

Sometimes, though, a diagnosis is enough. "There are tons of examples of patients who have gone through lots of testing for years and years, and you do this type of test and they get an answer," says Christian Marshall, a geneticist at the Hospital for Sick Children in Toronto, Canada. "It can end someone's diagnostic odyssey.”

\section{CRACKING THE CODE}

Scientists have been screening DNA since the 1960s for a growing list of genetic abnormalities that have been linked with disorders such as phenylketonuria, Down's syndrome and cystic fibrosis. But the complete sequencing of the human genome in 2003 raised an exciting new possibility. Instead of looking for specific mutations that are known to cause symptoms, scientists can read all of an individual's DNA and look for abnormalities. By comparing a person's pattern of A, T, C and G nucleotides 
with typical sequences from healthy individuals, they can find previously unknown anomalies in the code that might be linked to symptoms that have not been explained by conventional genetic tests.

That dream has become increasingly realistic as sequencing has got much faster and more affordable (see page S54). People such as Davis who have long-undiagnosed diseases have been among the first to benefit from sequencing, which can solve genetic mysteries about $30 \%$ of the time.

Sequencing is particularly valuable in the diagnosis of genetic forms of vision loss, neurodevelopmental delays, and cases of acute illness in children. Success rates are also higher when both of a patient's parents are sequenced, ranging from $40 \%$ to nearly $75 \%$.

In some patients, sequencing far outperforms older forms of genetic analysis, says Marshall. Of 100 children referred for genetic testing, he found that whole-genome sequencing produced accurate diagnoses in $34 \%$ - a dramatic improvement on the $13 \%$ diagnosed by the standard chromosomal microarray analysis and targeted panel sequencing.

Around the world, an estimated 350 million people suffer from rare diseases, according to Global Genes, a US-based patient advocacy organization. Half of them are children, and about $80 \%$ have a genetic basis. So far, hundreds of thousands of exomes and genomes have been sequenced, and thousands of people have been diagnosed this way, says Heidi Rehm, a molecular geneticist at Harvard Medical School in Boston, Massachusetts.

Sometimes, she says, diagnoses lead to lifechanging and even life-saving treatments. The information reverberates through families, prompting relatives to seek treatment or to alter their behaviour to prevent the onset of conditions identified this way. When doctors find multiple unrelated people with the same rare disease, they are also increasingly able to define new disorders, helping subsequent patients to find answers more quickly.

"Genome-scale sequencing has offered a truly tremendous leap in our ability to diagnose diseases that have at their root a genetic cause," Evans says. "I'm in the position of saying all the time to patients who have been looking for a diagnosis for years that we found it."

\section{MEANING IN MUTATIONS}

Despite the success stories, as many as $75 \%$ of patients with suspected genetic disorders fail to get answers, even after sequencing. One reason is that being able to read the code is not enough - the hard part is interpreting it. Different groups often provide conflicting analyses of the same genome. In one recent study, nine diagnostic laboratories analysed 99 genetic abnormalities. Tellingly, the labs agreed on results only one-third of the time. After discussion and review, the level of agreement rose, but only to $71 \%$.

One reason why consensus is so elusive is that genetic variation is remarkably common. A typical genome contains millions of variants that may or may not affect health. Some disorders involve multiple genetic variations working together, and these continue to elude search algorithms, says Matthew Hurles, a geneticist at the Wellcome Trust Sanger Institute in Hinxton, UK. Hurles also leads the Deciphering Developmental Disorders study, which is analysing the exomes of around 14,000 families of children with severe and undiagnosed illnesses. Even when a single mutation explains a disorder, establishing that connection requires statistical sleuthing, complex data analysis and clinical tests to provide confirmation, Hurles says.

To find meaning in the mutations, several major efforts are under way to compile large data sets and encourage open sharing - a departure from long-

"It helpsus standing efforts by some a lot to know there are other kids out there with the same things going on that Grace has." commercial and academic groups to keep their genetic discoveries secret and proprietary. The UK database DECIPHER, based at the Sanger Institute, has compiled 18,000 cases from 250 academic centres around the world, and has contributed to more than 1,000 published papers.

In the United States, the Clinical Genome Resource (ClinGen), which is funded by the National Institutes of Health, maintains another freely available database called ClinVar. The DECIPHER and ClinGen databases are now linked, along with other data sets, by a central hub called Matchmaker Exchange - a project that required three years just to build the infrastructure. "To make good use of the data, we had to invent a lot of informatics processes and procedures," Hurles says. "With big data comes big headaches."

Meanwhile, researchers are trying to figure out what to do when sequencing turns up potentially troubling variants that have nothing to do with the medical problem being investigated. Sequencing a child who has developmental delays, for example, might reveal a higher than normal risk of developing breast cancer or early-onset Alzheimer's disease. These secondary findings appear in around $1 \%$ of people sequenced, Rehm says, raising questions about how to share those results with patients, as well as concerns about traumatizing their families.

When genomicist Eric Schadt started teaching students to sequence genomes by doing their own, deans of other medical schools warned him that students might commit suicide if they discovered they were at high risk for certain diseases, or that their families might sue. Those fears failed to play out. "It has been a much more positive experience than a negative one," says Schadt, director of the Icahn Institute for Genomics and Multiscale Biology in New York. "Most people do want information and are able to handle it."

Physicians are also able to incorporate the technology into routine clinical care without assistance from medical geneticists, Rehm says. She is working on a project called Medseq, which is a randomized clinical trial that includes physicians and patients, and aims to develop reliable procedures for delivering sequencing results. Even when doctors are not experts in genetics, the study shows that they can understand the results and deliver them to patients. "The question has been whether physicians are going to order all sorts of tests and incorrectly interpret the information," Rehm says. "We haven't really found that."

\section{BEYOND THE DATA}

Turning diagnoses into treatments remains a work in progress, however. Most patients who get answers find themselves in a state of limbo with a new-found disease that nobody knows how to treat or cure. Nevertheless, many patients say it still brings them a degree of peace, especially after years spent bouncing from doctor to doctor. With a molecular diagnosis, patients get validation and a sense of closure. They may learn whether their mutations were inherited or acquired spontaneously, providing valuable information for their families. And a label helps them find new communities.

Sophia Mercer, a student and stay-at-home mother in Raleigh, North Carolina, experienced a huge sense of relief in 2012 when a sequencing study at the University of North Carolina revealed that her five-year-old daughter, Grace, had a mutation in a gene called DYRK1A. After years of tests and false diagnoses, the results finally explained what caused Grace's mental and physical disabilities, which include microcephaly and global developmental delays.

Now aged nine, Grace still wears nappies. She has trouble listening at school. When startled, she runs away with her hands over her ears and no concern for her safety. Knowing what's happening at the genetic level has helped her doctors and teachers rule out other conditions, such as ADHD. The diagnosis also relieved Mercer of guilt, as she was sure she must have done something while pregnant to cause Grace's problems. Through Facebook, she has found an international support group of families with DYRK1A mutations.

The family now feels much less alone. "It helps us a lot to know there are other kids out there with the same things going on that Grace has," says Mercer. "We were able to pinpoint specifically what was going on with our daughter. That in itself is a miracle."

Emily Sohn is a freelance journalist based in Minneapolis, Minnesota. 